\title{
Coincidence points of self mappings and left total relations
}

\author{
Laila A. Alnaser ${ }^{a}$, Durdana Lateef ${ }^{a}$, Hoda A. Fouad ${ }^{a, b}$, Jamshaid Ahmad ${ }^{c, *}$ \\ ${ }^{a}$ Department of Mathematics, College of Science, Taibah University, Al Madina Al Munawara, 41411, Kingdom of Saudi Arabia. \\ ${ }^{b}$ Department of Mathematics and Computer Science, Faculty of Science, Alexandria University, Alexandria, Egypt. \\ ${ }^{c}$ Department of Mathematics, University of Jeddah, P. O. Box 80327, Jeddah 21589, Saudi Arabia.
}

\begin{abstract}
The aim of this article is to prove some coincidence and fixed point theorems of hybrid contractions involving left total relations and single-valued mappings in the setting of $\mathcal{F}$-metric spaces which was first introduced by Jleli and Samet [M. Jleli, B. Samet, J. Fixed Point Theory Appl., 20 (2018), 20 pages]. Finally, an example is also presented to verify the effectiveness and applicability of our main results.
\end{abstract}

Keywords: $\mathcal{F}$-metric space, fixed point, rational contraction.

2010 MSC: 47H10, 54H 25.

(C)2019 All rights reserved.

\section{Introduction and preliminaries}

Over the past few decades, fixed point theory become a distinguished mathematical theory which is a pretty mixture of analysis, topology, and geometry. It is an interdisciplinary theory in which the existence of linear and nonlinear problems is frequently transformed into fixed point problems, for example, the existence of solutions to partial differential equations, the existence of solutions to integral equations, and the existence of periodic orbits in dynamical systems. This makes fixed point theory a contemporary area and a subject of active scientific research, constantly evolving and growing in a perpetual progress.

Banach's contraction principle [2] is one of the pivotal results of fixed point theory, which establishes that, if $F$ is a mapping from a complete metric space $(X, d)$ into itself and there exists a constant $k \in[0,1)$ such that

$$
d(F x, F y) \leqslant k d(x, y)
$$

for all $x, y \in X$, then $F$ has a unique fixed point in $X$.

In 2012, Azam [1] obtained some coincidence points of self mappings and left total relations under generalized contractive conditions in a metric spaces.

\footnotetext{
*Corresponding author

Email addresses: alnaser_layla@yahoo.com (Laila A. Alnaser), drdurdanamaths@gmail.com (Durdana Lateef),

hoda_rg@yahoo.com (Hoda A. Fouad), jkhan@uj.edu.sa (Jamshaid Ahmad)

doi: $10.22436 /$ jnsa.012.09.03
}

Received: 2019-01-25 Revised: 2019-02-21 Accepted: 2019-04-04 
Let $S_{1}$ and $S_{2}$ be arbitrary nonempty sets. A relation $R$ from $S_{1}$ to $S_{2}$ is a subset of $S_{1} \times S_{2}$ and is denoted by $R: S_{1} \rightarrow S_{2}$. The statement $(x, y) \in R$ is read " $x$ is R-related to $y$ ", and is denoted by $x R y$. A relation $R: S_{1} \rightarrow S_{2}$ is called left-total, if for all $x \in S_{1}$ there exists a $y \in S_{2}$ such that $x$ Ry that is $R$ is a multivalued function. A relation $R: S_{1} \rightarrow S_{2}$ is called right-total, if for all $y \in S_{2}$ there exists an $x \in S_{1}$ such that $x$ Ry. A relation $R: S_{1} \rightarrow S_{2}$ is known as functional, if $x R y, x R z$ implies that $y=z$, for $x \in S_{1}$ and $y, z \in S_{2}$. A mapping $F: S_{1} \rightarrow S_{2}$ is a relation from $S_{1}$ to $S_{2}$ which is both functional and left-total.

For $R: S_{1} \rightarrow S_{2}, J \subset S_{1}$ we define

$$
\begin{aligned}
R(J) & =\left\{b \in S_{2}: a R b \text { for some } x \in J\right\}, \\
\operatorname{dom}(R) & =\left\{a \in S_{1}: R(\{a\}) \neq \phi\right\}, \\
\operatorname{Range}(R) & =\left\{b \in S_{2}: b \in R(\{a\}) \text { for some } a \in \operatorname{dom}(R)\right\} .
\end{aligned}
$$

For convenience, we denote $R(\{a\})$ by $R\{a\}$. The class of relations from $S_{1}$ to $S_{2}$ is denoted by $\mathcal{R}\left(S_{1}, S_{2}\right)$. Thus the collection $\mathcal{M}\left(S_{1}, S_{2}\right)$ of all mappings from $S_{1}$ to $S_{2}$ is a proper sub collection of $\mathcal{R}\left(S_{1}, S_{2}\right)$. An element $x^{*} \in S_{1}$ is called coincidence point of $F: S_{1} \rightarrow S_{2}$ and $R: S_{1} \rightarrow S_{2}$ if $F x^{*} \in R\left\{x^{*}\right\}$. In the following we always suppose that $X$ is nonempty set and $(Y, d)$ is a metric space. For $R: X \rightarrow Y$ and $a, b \in \operatorname{dom}(R)$, we define

$$
D(R\{a\}, R\{b\})=\inf _{a R x, b R y} d(x, y) .
$$

The author [1] gave the following results as a consequence of his main result.

Theorem 1.1 ([1]). Let $\mathrm{X}$ be a nonempty set and $(\mathrm{Y}, \mathrm{d})$ be a metric space. Let $\mathrm{F}: \mathrm{X} \rightarrow \mathrm{Y}$ be single-valued mapping, $\mathrm{R}: \mathrm{X} \rightarrow \mathrm{Y}$ be such that $\mathrm{R}$ is left-total, Range $(\mathrm{F}) \subseteq \operatorname{Range}(\mathrm{R})$ and Range $(\mathrm{F})$ or Range $(\mathrm{R})$ is complete. If there exists a constant $\mathrm{k} \in[0,1)$ such that

$$
d(F x, F y) \leqslant k D(R\{x\}, R\{y\}),
$$

for all $x, y \in X$. Then there exists $w \in X$ such that $F w \in R\{w\}$.

Theorem $1.2([1])$. Let $\mathrm{X}$ be a nonempty set and $(\mathrm{Y}, \mathrm{d})$ be a metric space. Let $\mathrm{F}, \mathrm{R}: \mathrm{X} \rightarrow \mathrm{Y}$ be two mappings such that Range $(\mathrm{F}) \subseteq$ Range $(\mathrm{R})$ and Range $(\mathrm{F})$ or Range $(\mathrm{R})$ is complete. If there exists a constant $\mathrm{k} \in(0,1)$ such that

$$
\mathrm{D}(\mathrm{Fx}, \mathrm{Fy}) \leqslant \mathrm{kD}(\mathrm{Rx}, \mathrm{Ry}),
$$

for all $x, y \in X$. Then $F$ and $R$ have a coincidence point in $X$. Moreover, if either $F$ or $\mathrm{R}$ is injective, then $\mathrm{R}$ and $\mathrm{F}$ have a unique coincidence point in $\mathrm{X}$.

Very recently, Jleli and Samet [5] introduced an interesting generalization of a metric space in the following way.

Let $\mathcal{F}$ be the set of continuous functions $f:(0,+\infty) \rightarrow \mathbb{R}$ satisfying the following conditions:

$\left(\mathcal{F}_{1}\right) \mathrm{f}$ is non-decreasing, i.e., $0<\mathrm{s}<\mathrm{t}$ implies $\mathrm{f}(\mathrm{s}) \leqslant \mathrm{f}(\mathrm{t})$;

$\left(\mathcal{F}_{2}\right)$ For every sequence $\left\{t_{n}\right\} \subseteq R^{+}, \lim _{n \rightarrow \infty} \alpha_{n}=0$ if and only if $\lim _{n \rightarrow \infty} f\left(\alpha_{n}\right)=-\infty$.

Definition 1.3 ([5]). Let $X$ be a nonempty set, and let $D: X \times X \rightarrow[0,+\infty)$ be a given mapping. Suppose that there exists $(f, \alpha) \in \mathcal{F} \times[0,+\infty)$ such that

$\left(D_{1}\right)(x, y) \in X \times X, D(x, y)=0$ if and only if $x=y$;

$\left(D_{2}\right) D(x, y)=D(y, x)$, for all $(x, y) \in X \times X$;

$\left(D_{3}\right)$ For every $(x, y) \in X \times X$, for every $N \in \mathbb{N}, N \geqslant 2$, and for every $\left(u_{i}\right)_{i=1}^{N} \subset X$, with $\left(u_{1}, u_{N}\right)=(x, y)$, we have

$$
D(x, y)>0 \Rightarrow f(D(x, y)) \leqslant f\left(\sum_{i=1}^{N-1} D\left(x_{i}, x_{i+1}\right)\right)+\alpha
$$


Then $\mathrm{D}$ is said to be an $\mathcal{F}$-metric on $X$, and the pair $(X, D)$ is said to be an $\mathcal{F}$-metric space.

Remark 1.4. They showed that any metric space is an $\mathcal{F}$-metric space but the converse is not true in general, which confirms that this concept is more general than the standard metric concept.

Example 1.5 ([5]). The set of real numbers $\mathbb{R}$ is an $\mathcal{F}$-metric space, if we define $\mathrm{D}$ by

$$
D(x, y)= \begin{cases}(x-y)^{2}, & \text { if }(x, y) \in[0,3] \times[0,3] \\ |x-y|, & \text { if }(x, y) \notin[0,3] \times[0,3]\end{cases}
$$

with $f(t)=\ln (t)$ and $\alpha=\ln (3)$.

Definition $1.6([5])$. Let $(X, D)$ be an $\mathcal{F}$-metric space.

(i) Let $\left\{x_{n}\right\}$ be a sequence in $X$. We say that $\left\{x_{n}\right\}$ is $\mathcal{F}$-convergent to $x \in X$ if $\left\{x_{n}\right\}$ is convergent to $x$ with respect to the $\mathcal{F}$-metric $D$.

(ii) A sequence $\left\{x_{n}\right\}$ is $\mathcal{F}$-Cauchy, if

$$
\lim _{n, m \rightarrow \infty} D\left(x_{n}, x_{m}\right)=0 .
$$

(iii) We say that $(X, D)$ is $\mathcal{F}$-complete, if every $\mathcal{F}$-Cauchy sequence in $X$ is $\mathcal{F}$-convergent to a certain element in $X$.

Theorem 1.7 ([5]). Let (X, D) be an $\mathcal{F}$-metric space and $\mathrm{g}: \mathrm{X} \rightarrow \mathrm{X}$ be a given mapping. Suppose that the following conditions are satisfied:

(i) $(\mathrm{X}, \mathrm{D})$ is $\mathcal{F}$-complete;

(ii) there exists $k \in(0,1)$ such that

$$
\mathrm{D}(\mathrm{g}(\mathrm{x}), g(y)) \leqslant \mathrm{kD}(x, y) .
$$

Then $g$ has a unique fixed point $x^{*} \in X$. Moreover, for any $x_{0} \in X$, the sequence $\left\{x_{n}\right\} \subset X$ defined by

$$
x_{n+1}=g\left(x_{n}\right), n \in \mathbb{N},
$$

is $\mathcal{F}$-convergent to $x^{*}$.

Afterward, Hussain et al. considered the notion of $\alpha-\psi$-contraction in the setting of $\mathcal{F}$-metric spaces and proved the following fixed point theorem.

Theorem 1.8 ([3]). Let (X,D) be an $\mathcal{F}$-metric space and $\mathrm{T}: \mathrm{X} \rightarrow \mathrm{X}$ be $\beta$-admissible mapping. Suppose that the following conditions are satisfied:

(i) $(\mathrm{X}, \mathrm{D})$ is $\mathcal{F}$-complete;

(ii) there exist two functions $\beta: X \times X \rightarrow[0,+\infty)$ and $\psi \in \Psi$ such that

$$
\beta(x, y) D(T(x), T(y)) \leqslant \psi(M(x, y)),
$$

where

$$
M(x, y)=\max \{D(x, y), D(x, T x), D(y, T y)\}
$$

for $x, y \in X$;

(iii) there exists $x_{0} \in X$ such that $\beta\left(x_{0}, T\left(x_{0}\right)\right) \geqslant 1$.

Then $\mathrm{T}$ has a unique fixed point $\mathrm{x}^{*} \in \mathrm{X}$.

In this paper, we prove some coincidence point theorems of left total relations and single-valued mappings in the context of $\mathcal{F}$-metric spaces and derive the results of Azam and Jleli and Samet. 


\section{Main results}

Now we state and prove our main results in this section.

Theorem 2.1. Let $\mathrm{X}$ be a nonempty set and $(\mathrm{Y}, \mathrm{D})$ be an $\mathcal{F}$-metric space. Let $\mathrm{F}: \mathrm{X} \rightarrow \mathrm{Y}$ be single-valued mapping, $\mathrm{R}: \mathrm{X} \rightarrow \mathrm{Y}$ be such that $\mathrm{R}$ is left-total, Range $(\mathrm{F}) \subseteq \operatorname{Range}(\mathrm{R})$ and $\operatorname{Range}(\mathrm{F})$ or Range $(\mathrm{R})$ is $\mathcal{F}$-complete. If there exists a constant $\mathrm{k} \in(0,1)$ such that

$$
D(F x, F y) \leqslant k D(R\{x\}, R\{y\}),
$$

for all $x, y \in X$, then there exists $w \in X$ such that $F w \in R\{w\}$.

Proof. Let $x_{0} \in X$ be an arbitrary, but fixed element. We define the sequences $\left\{x_{n}\right\} \subset X$ and $\left\{y_{n}\right\} \subset$ Range $(R)$. Let $y_{1}=F x_{0}$, since Range $(F) \subseteq \operatorname{Range}(R)$. We may choose $x_{1} \in X$ such that $x_{1} R y_{1}$, since $R$ is left-total. Let $y_{2}=F x_{1}$, since Range $(F) \subseteq \operatorname{Range}(R)$. If $F x_{0}=F x_{1}$, then we have $x_{1} R y_{2}$. This implies that $x_{1}$ is the required point that is $F x_{1} \in R\left\{x_{1}\right\}$. So we assume that $F x_{0} \neq F x_{1}$, then from (2.1) we get

$$
\mathrm{D}\left(\mathrm{y}_{1}, \mathrm{y}_{2}\right)=\mathrm{D}\left(\mathrm{F} \mathrm{x}_{0}, \mathrm{~F} \mathrm{x}_{1}\right) \leqslant \mathrm{kD}\left(\mathrm{R}\left\{\mathrm{x}_{0}\right\}, \mathrm{R}\left\{\mathrm{x}_{1}\right\}\right) .
$$

We may choose $x_{2} \in X$ such that $x_{2} R y_{2}$, since $R$ is left-total. Let $y_{3}=F x_{2}$, since Range $(F) \subseteq R$ Range $(R)$. If $F x_{1}=F x_{2}$, then we have $x_{2} R y_{3}$. This implies that $F x_{2} \in R\left\{x_{2}\right\}$ and $x_{2}$ is the coincidence point. So $F x_{1} \neq F x_{2}$, then from (2.1), we get

$$
\mathrm{D}\left(\mathrm{y}_{2}, \mathrm{y}_{3}\right)=\mathrm{D}\left(\mathrm{F} \mathrm{x}_{1}, \mathrm{Fx} \mathrm{x}_{2}\right) \leqslant \mathrm{kD}\left(\mathrm{R}\left\{\mathrm{x}_{1}\right\}, \mathrm{R}\left\{\mathrm{x}_{2}\right\}\right) .
$$

By induction, we can construct sequences $\left\{x_{n}\right\} \subset X$ and $\left\{y_{n}\right\} \subset$ Range $(R)$ such that

$$
y_{n}=F x_{n-1} \quad \text { and } \quad x_{n} R y_{n},
$$

for all $n \in \mathbb{N}$. If there exists $n_{0} \in \mathbb{N}$ for which $F x_{n_{0}-1}=F x_{n_{0}}$. Then $x_{n_{0}} R y_{n_{0}+1}$. Thus $F x_{n_{0}} \in R\left\{x_{n_{0}}\right\}$ and the proof is finished. So we suppose now that $F x_{n-1} \neq F x_{n}$ for every $n \in \mathbb{N}$. Then from (2.2), (2.3) and (2.4), we deduce that

$$
\left.D\left(y_{n}, y_{n+1}\right)\right)=D\left(F x_{n-1}, F x_{n}\right) \leqslant k D\left(R\left\{x_{n-1}\right\}, R\left\{x_{n}\right\}\right),
$$

for all $n \in \mathbb{N}$. Since $x_{n} R y_{n}$ and $x_{n+1} R y_{n+1}$, therefore by the definition of $D$ for left total relation, we get $D\left(R\left\{x_{n-1}\right\}, R\left\{x_{n}\right\}\right) \leqslant D\left(y_{n-1}, y_{n}\right)$. Thus from (2.5), we have

$$
D\left(y_{n}, y_{n+1}\right) \leqslant k D\left(y_{n-1}, y_{n}\right),
$$

which further implies that

$$
D\left(y_{n}, y_{n+1}\right) \leqslant k D\left(y_{n-1}, y_{n}\right) \leqslant k^{2} D\left(y_{n-2}, y_{n-1}\right) \leqslant \ldots \leqslant k^{n} D\left(y_{0}, y_{1}\right) .
$$

It further yields that

$$
\sum_{i=n}^{m-1} D\left(y_{i}, y_{i+1}\right) \leqslant \frac{k^{n}}{1-k} D\left(y_{0}, y_{1}\right), \quad m>n .
$$

Since

$$
\lim _{n \rightarrow \infty} \frac{k^{n}}{1-k} D\left(y_{0}, y_{1}\right)=0
$$

there exists some $N \in \mathbb{N}$ such that

$$
0<\frac{k^{n}}{1-k} D\left(y_{0}, y_{1}\right)<\delta, \quad n \geqslant N .
$$

Next, let $(f, \alpha) \in F \times[0,+\infty)$ be such that $\left(D_{3}\right)$ is satisfied. Let $\epsilon>0$ be fixed. By $\left(\mathcal{F}_{2}\right)$, there exists $\delta>0$ such that

$$
0<\mathrm{t}<\delta \Longrightarrow \mathrm{f}(\mathrm{t})<\mathrm{f}(\delta)-\alpha
$$


Hence, by (2.6), (2.7) and $\left(\mathcal{F}_{2}\right)$, we have

$$
f\left(\sum_{i=n}^{m-1} D\left(y_{i}, y_{i+1}\right)\right) \leqslant f\left(\frac{k^{n}}{1-k} D\left(y_{0}, y_{1}\right)\right)<f(\epsilon)-\alpha,
$$

for $m>n \geqslant N$. Using $\left(D_{3}\right)$ and (2.5), we obtain $D\left(y_{n}, y_{m}\right)>0, m>n \geqslant N$ implies

$$
f\left(D\left(y_{n}, y_{m}\right)\right) \leqslant f\left(\sum_{i=n}^{m-1} D\left(y_{i}, y_{i+1}\right)\right)+\alpha<f(\epsilon),
$$

which implies by $\left(\mathcal{F}_{1}\right)$ that $D\left(y_{n}, y_{m}\right)<\epsilon, m>n \geqslant N$. This proves that $\left\{y_{n}\right\}$ is $\mathcal{F}$-Cauchy in Range $(R)$. Since Range $(R)$ is $\mathcal{F}$-complete, there exist $z \in \operatorname{Range}(R)$ such that $\left\{y_{n}\right\}$ is $\mathcal{F}$-convergent to $z$, i.e.,

$$
\lim _{n \rightarrow \infty} \mathrm{D}\left(\mathrm{y}_{n}, z\right)=0 \text {. }
$$

Now since $\mathrm{R}$ is left-total, so $w \mathrm{Rz}$ for some $w \in \mathrm{X}$. Now

$$
\begin{aligned}
\mathrm{D}\left(y_{n}, \mathrm{Fw}\right) & =\mathrm{D}\left(\mathrm{F} x_{n-1}, F w\right) \leqslant k D\left(R\left\{x_{n-1}\right\}, R\{w\}\right) \\
& \leqslant k D\left(y_{n-1}, z\right) .
\end{aligned}
$$

By $\left(D_{3}\right)$, we have

$$
\begin{aligned}
f(D(z, F w)) & \leqslant f\left(D\left(z, y_{n}\right)+D\left(y_{n}, F w\right)\right)+\alpha \\
& =f\left(D\left(z, y_{n}\right)+D\left(F x_{n-1}, F w\right)\right)+\alpha, n \in N .
\end{aligned}
$$

By $(2.1)$ and $\left(\mathcal{F}_{1}\right)$, we obtain

$$
\begin{aligned}
f(D(z, F w)) & \leqslant f\left(D\left(z, y_{n}\right)+k D\left(R\left\{x_{n-1}\right\}, R\{w\}\right)\right)+\alpha \\
& \leqslant f\left(D\left(z, y_{n}\right)+k D\left(y_{n-1}, z\right)\right)+\alpha, n \in N
\end{aligned}
$$

for $n \in N$. On the other hand, using $\left(\mathcal{F}_{2}\right)$ and $(2.8)$, we have

$$
\lim _{n \rightarrow \infty} f\left(D\left(z, y_{n}\right)+k D\left(y_{n-1}, z\right)\right)+\alpha=-\infty,
$$

which is a contradiction. Therefore, we have $d(z, F w)=0$, i.e., $z=F w$. Hence $F w \in R\{w\}$. In the case when Range $(F)$ is $\mathcal{F}$-complete. Since Range $(F) \subseteq \operatorname{Range}(R)$, so there exists an element $z^{*} \in \operatorname{Range}(R)$ such that $y_{n} \rightarrow z^{*}$. The remaining part of the proof is same as in previous case.

Example 2.2. Let $X=Y=\mathbb{R}$ endowed with $\mathcal{F}$-complete $\mathcal{F}$-metric $D$ given by

$$
D(x, y)= \begin{cases}\exp (|x-y|), & \text { if } x \neq y \\ 0, & \text { if } x=y\end{cases}
$$

Take $f(t)=\frac{-1}{t}$ and $\alpha=1$. Define $F: X \rightarrow Y$ as

$$
\mathrm{Fx}= \begin{cases}0 & \text { if } x \in \mathbb{Q}^{\prime} \\ 1 & \text { if } x \in \mathbb{Q}^{\prime}\end{cases}
$$

and $R: X \rightarrow Y$

$$
\mathrm{R}=(\mathbb{Q} \times[0,3]) \cup\left(\mathbb{Q}^{\prime} \times[7,9]\right) .
$$

Then Range $(F)=\{0,1\} \subset$ Range $(R)=[0,3] \cup[7,9]$. For $x \in \mathbb{Q}, y \in \mathbb{Q}^{\prime}$ or either $y \in \mathbb{Q}, x \in \mathbb{Q}^{\prime}$, we have $\mathrm{d}(\mathrm{Fx}, \mathrm{Fy}) \neq 0$ implies

$$
D(F x, F y)) \leqslant k D(R\{x\}, R\{y\})),
$$

with $k=\frac{1}{2}$. Thus all conditions of the above theorem are satisfied and 1 is the coincidence point of $F$ and $\mathrm{R}$. 
From Theorem 2.1, we deduce the following result immediately.

Theorem 2.3. Let $\mathrm{X}$ be a nonempty set and $(\mathrm{Y}, \mathrm{D})$ be an $\mathcal{F}$-metric space. Let $\mathrm{F}, \mathrm{R}: \mathrm{X} \rightarrow \mathrm{Y}$ be two mappings such that Range $(F) \subseteq$ Range $(R)$ and Range $(F)$ or Range $(R)$ is $\mathcal{F}$-complete. If there exists a constant $k \in(0,1)$ such that

$$
\mathrm{D}(\mathrm{Fx}, \mathrm{Fy}) \leqslant \mathrm{kD}(\mathrm{Rx}, \mathrm{Ry}),
$$

for all $x, y \in X$, then $F$ and $R$ have a coincidence point in $X$. Moreover, if either $F$ or $R$ is injective, then $R$ and $F$ have a unique coincidence point in $\mathrm{X}$.

Proof. By Theorem 2.1, we obtain that there exists $w \in X$ such that $F w=R w$, where

$$
R w=\lim _{n \rightarrow \infty} R x_{n}=\lim _{n \rightarrow \infty} F x_{n-1}, \quad x_{0} \in X
$$

For uniqueness, assume that $w_{1}, w_{2} \in X, w_{1} \neq w_{2}, F w_{1}=R w_{1}$ and $F w_{2}=R w_{2}$. Then

$$
\left.\mathrm{D}\left(\mathrm{F} \mathfrak{w}_{1}, \mathrm{~F} \mathfrak{w}_{2}\right)\right) \leqslant k \mathrm{D}\left(\mathrm{R} w_{1}, \mathrm{R} w_{2}\right),
$$

for any $k \in(0,1)$. If $R$ or $F$ is injective, then

$$
\left.\mathrm{D}\left(\mathrm{R} w_{1}, \mathrm{R} w_{2}\right)\right)=\mathrm{D}\left(\mathrm{F} w_{1}, \mathrm{~F} w_{2}\right) \leqslant \mathrm{kD}\left(\mathrm{R} w_{1}, \mathrm{R} w_{2}\right)<\mathrm{D}\left(\mathrm{R} w_{1}, \mathrm{R} w_{2}\right),
$$

a contradiction. Thus proved.

Corollary 2.4 ([4]). Let (X,D) be an $\mathcal{F}$-metric space and $\mathrm{F}: \mathrm{X} \rightarrow \mathrm{X}$ be a self mapping. Suppose that the following conditions are satisfied:

(i) $(\mathrm{X}, \mathrm{D})$ is $\mathcal{F}$-complete;

(ii) there exists a constant $\mathrm{k} \in(0,1)$ such that,

$$
D(F x, F y)) \leqslant k D(x, y)
$$

for all $x, y \in X$.

Then $\mathrm{F}$ has a unique fixed point.

Proof. Choosing $\mathrm{X}=\mathrm{Y}$ and $\mathrm{R}=\mathrm{I}$ (the identity mapping on $\mathrm{X}$ ).

By Remark 1.4, we can deduces several well-known fixed point theorems of the existing literature from our main results as special cases.

(1) Theorem 1.1 of Azam [1] from Theorem 2.1.

(2) Theorem 1.2 of Azam [1] from Theorem 2.3.

(3) The classical Banach contraction principle [2] from Corollary 2.4.

\section{Conclusion}

In this paper, we obtained some coincidence and fixed point theorems of hybrid contractions involving left total relations and single-valued mappings in the context of $\mathcal{F}$-metric spaces. In this way, we generalize several results of literature including the main result of Jleli et al. [5]. We also provided an example to verify the effectiveness and applicability of our main results. 


\section{Acknowledgment}

This project was funded by Deanship of Scientific Research (DSR), Taibah University, Al Madina Al Munawara, Kingdom of Saudi Arabia, under Grant No. 60348/1439. The authors are thankful to DSR for providing research facilities and financial support.

\section{References}

[1] A. Azam, Coincidence points of mappings and relations with applications, Fixed Point Theory Appl., 2012 (2012), 9 pages. 1, 1.1, 1.2, 2

[2] S. Banach, Sur les opérations dans les ensembles abstraits et leur applications aux équations intégrales, Fundam. Math., 3 (1922), 133-181. 1, 2

[3] A. Hussain, T. Kanwal, Existence and uniqueness for a neutral differential problem with unbounded delay via fixed point results, Trans. A. Razmadze Math. Inst., 172 (2018), 481-490. 1.8

[4] M. Jleli, B. Samet, A new generalization of the Banach contraction principle, J. Inequal. Appl. 2014 (2014), 8 pages. 2.4

[5] M. Jleli, B. Samet, On a new generalization of Metric Spaces, J. Fixed Point Theory Appl., 20 (2018), 20 pages. 1, 1.3, $1.5,1.6,1.7,3$ 